\title{
Basic theory and stability analysis for neutral stochastic functional differential equations with pure jumps
}

\author{
Mengling $\mathrm{Li}^{1}$, Feiqi Deng ${ }^{*} \&$ Xuerong $\mathrm{Mao}^{2}$ \\ ${ }^{1}$ Systems Engineering Institute, South China University of Technology, Guangzhou 510640, China; \\ ${ }^{2}$ Department of Statistics and Modelling Science, University of Strathclyde, Glasgow G1 1XH, UK \\ Received ; accepted
}

\begin{abstract}
This paper investigates the existence and uniqueness of solution of the neutral stochastic functional differential equations with pure jumps (NSFDEwPJs) as well as the boundedness and almost sure exponential stability. Generally, the classical existence and uniqueness theorem of solution can be obtained under the local Lipschitz condition and the linear growth condition. But there are many equations which do not obey the linear growth condition. Therefore, our first aim is to establish new theorems where the linear growth condition is no longer needed while the up-bound for the diffusion operator will play a leading role. Moreover, we can also obtain the boundedness of $p$ th moment of the solution and almost sure exponential stability of the trivial solution under some loose conditions. Finally, we will give two examples to illustrate the effectiveness of our results.
\end{abstract}

Keywords neutral stochastic functional differential equations, pure jumps, the existence and uniqueness theorem, stability analysis

Citation Author A, Author B, Author C, et al. Title for citation. Sci China Inf Sci, 2016, 59(1): xxxxxx, doi: xxxxxxxxxxxxxx

\section{Introduction}

As we know, many real systems will be affected by stochastic factors. So it is necessary for us to consider the stochastic systems. Of course, there is an extensive literature in this area such as [1-3]. Delayed systems are suited for describing such systems which do not only depend on the present states but also the past ones. In this paper we study a class of time delay systems depending on past and present values but that involves derivatives with delays as well as the function itself. Such systems historically have been referred to as neutral systems. Moreover, with the development of the stochastic analysis and the requirements of applications, Poisson jumps are considered by many researchers, see $[4,6]$. Moreover, there are practical applications for Poisson jump, such that financial market [5]. In this paper, we consider the neutral stochastic functional differential equations containing Poisson jumps which are complex equations. So we have to employ various stochastic analysis tools since we discuss this type of complicated equation under relatively loose conditions.

* Corresponding author (email: aufqdeng@scut.edu.cn) 
Our paper is motivated by the reference [7], they studied the existence and uniqueness of the solutions to NSFDEwPJs whose coefficients satisfy the local Lipschitz condition and the linear growth condition. However, the coefficients of many important equations such as stochastic delay Lotka-Volterra equations [8] do not satisfy the linear growth condition. So if we could find a wider condition to replace the linear growth condition, then many problems would be solved. Fortunately, we finally find a more general test for NSFDEwPJs that covers a wide class of highly nonlinear NSFDEwPJs. Here, we refer to the literatures [9-12] which also allow that there are high-order terms consisting in their parameters. But the above papers only focus on the stochastic models containing the continuous Brown motion. So far as we know, the stochastic models containing Poisson jump have not been investigated.

Based on the above discussion, our first result mainly utilizes the up-bounds for the diffusion operator to replace the linear growth condition, hence we can also obtain a unique global solution even if the coefficients of the equation are of highly order. In addition to this, in Section 4, we also utilize the modified conditions to deal with the asymptotic moment estimation which is also one of our main results. And moreover, if there exists a trivial solution for an equation, then the trivial solution will be almost surely exponentially stable. At last, we give two examples to illustrate the effectiveness of our theory.

\section{Preliminaries}

Throughout this paper, unless otherwise specified, we use the following notations. Let $\left(\Omega, \mathcal{F},\left\{\mathcal{F}_{t}\right\}_{t \geqslant 0}, \mathcal{P}\right)$ be a complete probability space with a filtration $\left\{\mathcal{F}_{t}\right\}_{t \geqslant 0}$ satisfying the usual conditions (i.e, it is right continuous and $\mathcal{F}_{0}$ contains all $\mathcal{P}$-null sets). If $A$ is a vector or matrix, $A^{T}$ denotes its transpose and its trace norm is denoted by $|A|=\sqrt{\operatorname{trace}\left(\mathrm{A}^{\mathrm{T}} \mathrm{A}\right)}$. Let $\tau>0$, and denote by $D\left([-\tau, 0] ; R^{n}\right)$ the family of all right-continuous functions with left limits $\phi$ from $[-\tau, 0]$ to $R^{n}$ equipped with the norm $\|\phi\|=$ $\sup _{-\tau \leqslant s \leqslant 0}|\phi(s)| . \quad D_{\mathcal{F}_{0}}^{b}$ denotes the family of all almost surely bounded, $\mathcal{F}_{0}$-measurable, $D\left([-\tau, 0] ; R^{n}\right)$ valued random variable $\xi=\{\xi(\theta):-\tau \leqslant \theta \leqslant 0\} . E(x)$ denotes the mathematical expectation of random variable $x$. Let $p \geqslant 2$, denoted by $\mathcal{L}_{\mathcal{F}_{0}}^{p}\left([-\tau, 0] ; R^{n}\right)$ the family of all $\mathcal{F}_{0}$-measurable, $D\left([-\tau, 0] ; \mathbb{R}^{n}\right)$ valued random variables $\phi=\{\phi(\theta):-\tau \leqslant \theta \leqslant 0\}$ such that $E \sup _{-\tau \leqslant \theta \leqslant 0}|\phi(\theta)|^{p}<\infty$. For $a, b \in$ $R, a \vee b$ (respectively, $a \wedge b$ ) means the maximum(respectively, minimum) of $a$ and $b$.

Let $(U, \mathcal{B}(U))$ be a measurable space and $\bar{p}(t)(t \geqslant 0)$ be the jump at time $t$. Then, for each Borel set $A \in \mathcal{B}(U-\{0\})$, the Poisson counting measure $N_{\bar{p}}$ is defined by

$$
N_{\bar{p}}(t, A):=\sum_{0<s \leqslant t} I_{A}(\bar{p}(s))=\#\{0<s \leqslant t, \bar{p}(s) \in A\},
$$

where $I(\cdot)$ denotes the indicative function and \# records the number of the jumps from 0 to $t$. According to [13], if we fix $t$ and $A$, then $N_{\bar{p}}(t, A)$ is a random variable. However, if we fix $\omega \in \Omega$ and $t \geqslant 0$, then $N_{\bar{p}}(t, \cdot)(\omega)$ is a measure. Therefore, if we fix $A$ with a Lévy measure $\pi(A)$, then $\left\{N_{\bar{p}}(t, A)\right\}_{t \geqslant 0}$ is a Poisson process with intensity $\pi(A)$. Moreover, we have

$$
P\left(N_{\bar{p}}(t, A)=n\right)=\frac{\exp (-\pi(A) t)(\pi(A) t)^{n}}{n !}
$$

and the measure $\tilde{N}_{\bar{p}}$ satisfying $\tilde{N}_{\bar{p}}(t, A)=N_{\bar{p}}(t, A)-\pi(A) t$ is a martingale measure.

Let $x(t)$ be an $R^{n}$-valued stochastic process on $t \in[-\tau, \infty)$ and $x_{t}=\{x(t+\theta):-\tau \leqslant \theta \leqslant 0\}$ for $t \geqslant 0$ be regarded as a $D\left([-\tau, 0] ; R^{n}\right)$-valued stochastic process. Consider the following NSFDEwPJs

$$
d\left[x(t)-D\left(x_{t}\right)\right]=f\left(t, x_{t}\right) d t+\int_{U} h\left(u, x_{t}\right) N_{\bar{p}}(d t, d u),
$$

where $f: R^{+} \times D\left([-\tau, 0] ; R^{n}\right) \rightarrow R^{n}$ and $h: U \times D\left([-\tau, 0] ; R^{n}\right) \rightarrow R^{n}$ are both Borel-measurable functions. In order to solve the equation we need to know the initial data, where we assume that the initial data is given by

$$
x_{0}=\xi=\{\xi(t):-\tau \leqslant t \leqslant 0\} \in \mathcal{L}_{\mathcal{F}_{0}}^{p}\left([-\tau, 0] ; R^{n}\right) .
$$


And $\tilde{N}_{\bar{p}}(d t, d u)=N_{\bar{p}}(d t, d u)-\pi(d u) d t$ is the compensated Poisson random measure.

The classical existence and uniqueness theorem requires the coefficients $f, h$ to satisfy the local Lipschitz condition and the linear growth condition and the neutral-term $D$ satisfies the contractility condition (see [7]). In this paper, we retain the contractility condition and the local Lipschitz condition but replace the linear growth condition by a more general condition. In order to state our condition, we need to introduce the well-known Lyapunov function and Itô formula.

Let $C^{1,2}\left(R^{+} \times R^{n} ; R^{+}\right)$denote the family of all nonnegative functions $V(t, x)$ on $R^{+} \times R^{n}$ which are continuously twice differentiable in $x$ and once in $t$, moreover, define

$$
\begin{aligned}
& V_{x}(t, x)=\left(\frac{\partial V(t, x)}{\partial x_{1}}, \ldots, \frac{\partial V(t, x)}{\partial x_{n}}\right), \\
& V_{x x}(t, x)=\left(\frac{\partial^{2} V(t, x)}{\partial x_{k} \partial x_{l}}\right)_{n \times n} . \\
& V_{t}(t, x)=\frac{\partial V(t, x)}{\partial t}
\end{aligned}
$$

Then, we can define an operator $\mathcal{L} V: R^{+} \times D\left([-\tau, 0] ; R^{n}\right) \rightarrow R$ for the function $V(t, x) \in C^{1,2}\left(R^{+} \times\right.$ $\left.R^{n} ; R^{+}\right)$by

$$
\begin{aligned}
\mathcal{L} V(t, \phi)= & V_{t}(t, \phi(0)-D(\phi))+V_{x}(t, \phi(0)-D(\phi)) f(t, \phi) \\
& +\int_{U}[V(t, \phi(0)-D(\phi)+h(u, \phi))-V(t, \phi(0)-D(\phi)) \\
& \left.-V_{x}(t, \phi(0)-D(\phi)) h(u, \phi)\right] \pi(d u),
\end{aligned}
$$

where $t \in R^{+}, \phi \in D\left([-\tau, 0], R^{n}\right)$.

Based on this, we can cite the Itô formula

$$
\begin{aligned}
d V\left(t, x(t)-D\left(x_{t}\right)\right)= & \mathcal{L} V\left(t, x_{t}\right) d t+\int_{U}\left[V\left(t, x(t)-D\left(x_{t}\right)+h\left(u, x_{t}\right)\right)\right. \\
& \left.-V\left(t, x(t)-D\left(x_{t}\right)\right)\right] \tilde{N}_{\bar{p}}(d t, d u) .
\end{aligned}
$$

We will need to make the following hypotheses.

Assumption 1. (Local Lipschitz condition) For arbitrary $\varphi, \psi \in D\left([-\tau, 0] ; R^{n}\right)$ and $\|\varphi\| \vee\|\psi\| \leqslant n$, there is a positive constant $k_{n}$ such that

$$
|f(\varphi, t)-f(\psi, t)|^{2} \vee \int_{U}|h(\varphi, u)-h(\psi, u)|^{2} \pi(d u) \leqslant k_{n}\|\varphi-\psi\|^{2},
$$

where $n \in N^{+}, t \in R^{+}, u \in U$. Moreover,

$$
L=\sup \{|f(t, 0)| \vee|h(u, 0)|: t \geqslant 0, u \in U\}<\infty .
$$

Assumption 2. There are three functions $V \in C^{2,1}\left(R^{n} \times[-\tau, \infty) ; R^{+}\right), H \in C\left(R^{n} \times[-\tau, \infty) ; R^{+}\right)$ and non-decreasing function $K(t) \in C\left(R^{+} ; R^{+}\right)$, two probability measure $\mu(\cdot)$ and $\nu(\cdot)$ as well as three positive constants $p(\geqslant 2), c_{1}, c_{2}$ such that

$$
c_{1}|x|^{p} \leqslant V(x, t) \leqslant c_{2}|x|^{p},
$$

for any $x \in R^{n}$ and

$$
\begin{aligned}
\mathcal{L} V(t, \phi) \leqslant & K(t)\left[1+V(t, \phi(0))+\int_{-\tau}^{0} V(t+\theta, \phi(\theta)) d \mu(\theta)\right] \\
& -H(t, \phi(0))+\int_{-\tau}^{0} H(t+\theta, \phi(\theta)) d \nu(\theta) .
\end{aligned}
$$


Assumption 3. (Contractility condition) $D(0)=0$ and there is a constant $k_{0} \in[0,1)$ such that

$$
E|D(\phi)|^{p} \leqslant k_{0}^{p} \sup _{-\tau \leqslant \theta \leqslant 0} E|\phi(\theta)|^{p},
$$

for all $\phi \in \mathcal{L}_{\mathcal{F}_{0}}^{p}\left([-\tau, 0] ; R^{n}\right)$.

In section 3 , the proof of our new existence and uniqueness theorem needs that there exists a maximal local solution for the equation (1) at first. So we should introduce the definition for maximal local solution and give a lemma about the existence of the maximal local solution.

Definition 1. (Maximal local solution) Let $0 \leqslant \sigma_{\infty} \leqslant T$ a.s. be a stopping time. An $\mathcal{F}_{t}$-adapted, $R^{n}$-valued, cadlag process $\left\{x(t):-\tau \leqslant t \leqslant \sigma_{\infty}\right\}$ (a cadlag process refers to a stochastic process which is right continuous and has left limit) is called a local solution to the equation (1) with initial data $x(t)=\xi(t)$ on $t \in[-\tau, 0]$ if for any stopping time $\sigma_{k} \leqslant \sigma_{\infty}$ and any $t \in[0, T]$

$$
x\left(t \wedge \sigma_{k}\right)-D\left(x_{t \wedge \sigma_{k}}\right)=\xi(0)-D(\xi)+\int_{0}^{t \wedge \sigma_{k}} f\left(x_{s}, s\right) d s+\int_{0}^{t \wedge \sigma_{k}} \int_{U} h\left(x_{s}, u\right) N_{\bar{p}}(d s, d u),
$$

holds with probability 1 . And, moreover if

$$
\lim _{t \rightarrow \sigma_{\infty}} \sup |x(t)|=\infty, \quad \text { whenever } \sigma_{\infty}<T,
$$

then $\{x(t)\}$ is called a maximal local solution of (1) and $\sigma_{\infty}$ is called the explosion time.

Lemma 1. If Assumptions 1,3 hold, then for any given initial data $\xi(t)$ on $[-\tau, 0]$, there exists a unique maximal local solution to equation (1). Proof. From Assumption 3, we have $D(\phi) \leqslant k_{0}\|\phi\|$. Let $k^{0} \in R^{+}$is sufficiently large for $\|\xi\| \leqslant k^{0}$. For any positive integer $k \geqslant k^{0}$, define

$$
z^{[k]}=\frac{|z| \wedge k}{|z|} z, \quad 0^{[k]}=0,
$$

for any $z \in R^{d}$. Then we can define the truncation functions

$$
f_{k}(t, y)=f\left(t, y^{[k]}\right), \quad h_{k}(u, y)=h\left(u, y^{[k]}\right),
$$

for $y \in R^{d}$.Next, we consider the following equation:

$$
d\left[x^{k}(t)-D\left(x_{t}^{k}\right)\right]=f_{k}\left(t, x_{t}^{k}\right) d t+\int_{U} h_{k}\left(u, x_{t}^{k}\right) N_{\bar{p}}(d t, d u),
$$

on $t \in[0, T]$ with initial data $x^{k}(t)=\xi(t)$ on $t \in[-\tau, 0]$. Then, we have

$$
\left|f_{k}(t, \phi)\right|^{2} \leqslant 2\left|f_{k}(t, \phi)-f(0, t)\right|^{2}+2 L \leqslant L_{1}\left(1+\|\phi\|^{2}\right),
$$

where $L_{1}=\max \left\{2 k_{k}, 2 L\right\}$. Similarly,

$$
\int_{U}\left|h_{k}(u, \phi)\right|^{2} \leqslant L_{1}\left(1+\|\phi\|^{2}\right) .
$$

By Assumption 1, the equation (4) satisfies the global Lipschitz condition and the linear growth condition. Therefore, according to the literature [7], there is a unique global solution $x^{k}(t)$ to $(4)$. Then, we define the stopping time

$$
\sigma_{k}=\inf \left\{t \in[0, T]:\left|x^{k}(t)\right| \geqslant k\right\},
$$

for $k \geqslant k_{0}$ and where we set $\inf \emptyset=\infty$ (as usual $\emptyset$ denotes the empty set) throughout our paper. Moreover, we can see that

$$
x^{k}(t)=x^{k+1}(t), \quad-\tau \leqslant t \leqslant \sigma_{k},
$$

which means that $\left\{\sigma_{k}\right\}$ is a nondecreasing sequence and then let $\lim _{k \rightarrow \sigma_{k}}=\sigma_{\infty}$ a.s. Consequently, we define $\left\{x(t):-\tau \leqslant t<\sigma_{\infty}\right\}$ with $x(t)=\xi(t)$ on $t \in[-\tau, 0]$ and

$$
x(t)=x^{k}(t), \quad t \in\left[\sigma_{k-1}, \sigma_{k}\right), k \geqslant 1,
$$


where $\sigma_{0}=0$. From (4) and (5), we can obtain

$$
x\left(t \wedge \sigma_{k}\right)-D\left(x_{t \wedge \sigma_{k}}\right)=\xi(0)-D(\xi)+\int_{0}^{t \wedge \sigma_{k}} f\left(x_{s}, s\right) d s+\int_{0}^{t \wedge \sigma_{k}} \int_{U} h\left(x_{s}, u\right) N_{\bar{p}}(d s, d u),
$$

for any $t \in[0, T]$. Moreover, if $\sigma_{\infty}<T$, then

$$
\lim _{t \rightarrow \sigma_{\infty}} \sup |x(t)| \geqslant \lim _{k \rightarrow \infty} \sup \left|x\left(\sigma_{k}\right)\right|=\lim _{k \rightarrow \infty} \sup \left|x_{k}\left(\sigma_{k}\right)\right|=\infty
$$

Hence $\left\{x(t):-\tau \leqslant t<\sigma_{\infty}\right\}$ is a maximal local solution to (1).

\section{The existence and uniqueness theorem}

In this section, we prove that there is a unique global solution to the equation (1) if the coefficients of the equation (1) satisfy the contractility condition, local Lipschitz condition and the Assumption 3.

Theorem 1. Let Assumptions 1,2,3 hold, then for given initial value $x_{0}=\xi \in C\left([-\tau, 0] ; R^{n}\right)$, there exists a unique global solution $x\left(t ; t_{0}, \xi\right)$ to equation $(1)$. Moreover, the solution has the property that

$$
E V(t, x(t)) \leqslant\left(0.5+c_{3}\right) e^{\frac{2 c_{2}}{c_{1}\left(1-k_{0}\right)^{p}} \int_{0}^{t} K(\rho+\tau) d \rho}
$$

where

$$
\begin{gathered}
c_{3}=\frac{c_{2} k_{0}}{1-k_{0}} E\|\xi\|^{p}+\frac{c_{2} M}{c_{1}\left(1-k_{0}\right)^{p}} \\
M=E\left(V\left(0, x(0)-D\left(x_{0}\right)\right)+K(\tau) \int_{-\tau}^{0} V(\rho, x(\rho)) d \rho+\int_{-\tau}^{0} H(\rho, x(\rho)) d \rho\right) .
\end{gathered}
$$

Proof. $\quad$ By Lemma 2.1, there is a unique maximal local solution $x(t)$ on $\left[-\tau, \sigma_{\infty}\right)$, where $\sigma_{\infty}$ is called the explosion time. Let $k^{0} \in R^{+}$be sufficiently large for $\|\xi\| \leqslant k^{0}$. For any integer $k \geqslant k^{0}$, define

$$
\tau_{k}=\inf \left\{t \in\left[0, \sigma_{\infty}\right):|x(t)| \geqslant k,\right\}
$$

where $\inf \emptyset=\infty$. Obviously, the sequence $\left\{\tau_{k}\right\}$ is increasing. So we have a limit $\tau_{\infty}=\lim _{k \rightarrow \infty} \tau_{k}$, whence $\tau_{\infty} \leqslant \sigma_{\infty}$. If we can show that $\tau_{\infty}=\infty$, then we have $\sigma_{\infty}=\infty$. Therefore, we only need to devote to prove $\tau_{\infty}=\infty$.

For any $s>0$, by Itô formula and Assumption 2, we have

$$
\begin{aligned}
V\left(s, x(s)-D\left(x_{s}\right)\right)= & V\left(0, x(0)-D\left(x_{0}\right)\right)+\int_{0}^{s} \mathcal{L} V\left(\rho, x_{\rho}\right) d \rho \\
& +\int_{0}^{s} \int_{U}\left[V\left(\rho, x(\rho)-D\left(x_{\rho}\right)+h\left(x_{\rho}, u\right)\right)-V\left(\rho, x(\rho)-D\left(x_{\rho}\right)\right)\right] \tilde{N}_{\bar{p}}(d \rho, d u) \\
& \leqslant V\left(0, x(0)-D\left(x_{0}\right)\right)+\int_{0}^{s} K(\rho)[1+V(\rho, x(\rho))] d \rho \\
& +\int_{0}^{s} \int_{-\tau}^{0} K(\rho) V(\rho+\theta, x(\rho+\theta)) d \mu(\theta) d \rho \\
& -\int_{0}^{s} H(\rho, x(\rho)) d \rho+\int_{0}^{s} \int_{-\tau}^{0} H(\rho+\theta, x(\rho+\theta)) d \nu(\theta) d \rho \\
& +\int_{0}^{s} \int_{U}\left[V\left(\rho, x(\rho)-D\left(x_{\rho}\right)+h\left(x_{\rho}, u\right)\right)-V\left(\rho, x(\rho)-D\left(x_{\rho}\right)\right)\right] \tilde{N}_{p}(d \rho, d u)
\end{aligned}
$$

At the same time, by the Fubini theorem and the fact that $K(t)$ is a non-decreasing function, thus we 
obtain

$$
\begin{aligned}
\int_{0}^{s} K(\rho) \int_{-\tau}^{0} V(\rho+\theta, x(\rho+\theta)) d \mu(\theta) d \rho & =\int_{-\tau}^{0} d \mu(\theta) \int_{0}^{s} K(\rho) V(\rho+\theta, x(\rho+\theta)) d \rho \\
\leqslant & \int_{-\tau}^{s} K(\rho+\tau) V(\rho, x(\rho)) d \rho \\
\leqslant & K(\tau) \int_{-\tau}^{0} V(\rho, x(\rho)) d \rho \\
& +\int_{0}^{s} K(\rho+\tau) V(\rho, x(\rho)) d \rho .
\end{aligned}
$$

Similarly, we have

$$
\int_{0}^{s} \int_{-\tau}^{0} H(\rho+\theta, x(\rho+\theta)) d \nu(\theta) d \rho \leqslant \int_{-\tau}^{s} H(\rho, x(\rho)) d \rho .
$$

Substituting (7) and (8) into (6) yields

$$
E V\left(s \wedge \tau_{k}, x\left(s \wedge \tau_{k}\right)-D\left(x_{s \wedge \tau_{k}}\right)\right) \leqslant M+E \int_{0}^{s \wedge \tau_{k}} K(\rho+\tau)[1+2 V(\rho, x(\rho))] d \rho,
$$

where $M=E\left(V\left(0, x(0)-D\left(x_{0}\right)\right)+K(\tau) \int_{-\tau}^{0} V(\rho, x(\rho)) d \rho+\int_{-\tau}^{0} H(\rho, x(\rho)) d \rho\right)$. Since $c_{1}|x|^{p} \leqslant V(x, t) \leqslant$ $c_{2}|x|^{p}$, by inequality (9), we have

$$
c_{1} E\left|x\left(s \wedge \tau_{k}\right)-D\left(x_{s \wedge \tau_{k}}\right)\right|^{p} \leqslant M+E \int_{0}^{s \wedge \tau_{k}} K(\rho+\tau)[1+2 V(\rho, x(\rho))] d \rho .
$$

Applying elementary inequality $|a+b|^{p} \leqslant(1-\varepsilon)^{1-p}|a|^{p}+\varepsilon^{1-p}|b|^{p}$ for any $a, b \in R$ and set $\varepsilon=k_{0}$, we have

$$
|x(s)|^{p} \leqslant\left(1-k_{0}\right)^{1-p}\left|x(s)-D\left(x_{s}\right)\right|^{p}+k_{0}^{1-p}\left|D\left(x_{s}\right)\right|^{p},
$$

which implies

$$
\begin{aligned}
E\left|x\left(s \wedge \tau_{k}\right)\right|^{p} & \leqslant\left(1-k_{0}\right)^{1-p} E\left|x\left(s \wedge \tau_{k}\right)-D\left(x_{s \wedge \tau_{k}}\right)\right|^{p}+k_{0}^{1-p} E\left|D\left(x_{s \wedge \tau_{k}}\right)\right|^{p} \\
& \leqslant\left(1-k_{0}\right)^{1-p} E\left|x\left(s \wedge \tau_{k}\right)-D\left(x_{s \wedge \tau_{k}}\right)\right|^{p}+k_{0} \sup _{-\tau \leqslant \theta \leqslant 0} E\left|x\left(s \wedge \tau_{k}+\theta\right)\right|^{p} \\
& \leqslant\left(1-k_{0}\right)^{1-p} E\left|x\left(s \wedge \tau_{k}\right)-D\left(x_{s \wedge \tau_{k}}\right)\right|^{p}+k_{0} E\|\xi\|^{p}+k_{0} \sup _{0 \leqslant u \leqslant s \wedge \tau_{k}} E|x(u)|^{p} \\
& \leqslant\left(1-k_{0}\right)^{1-p} E\left|x\left(s \wedge \tau_{k}\right)-D\left(x_{s \wedge \tau_{k}}\right)\right|^{p}+k_{0} E\|\xi\|^{p}+k_{0} \sup _{0 \leqslant u \leqslant s} E\left|x\left(u \wedge \tau_{k}\right)\right|^{p} .
\end{aligned}
$$

Then for any $t \geqslant s$, we have

$$
\begin{aligned}
\sup _{0 \leqslant s \leqslant t} E\left|x\left(s \wedge \tau_{k}\right)\right|^{p} \leqslant & \left(1-k_{0}\right)^{1-p} \sup _{0 \leqslant s \leqslant t} E\left|x\left(s \wedge \tau_{k}\right)-D\left(x_{s \wedge \tau_{k}}\right)\right|^{p} \\
& +k_{0} E\|\xi\|^{p}+k_{0} \sup _{0 \leqslant s \leqslant t} E\left|x\left(s \wedge \tau_{k}\right)\right|^{p}
\end{aligned}
$$

which implies

$$
\sup _{0 \leqslant s \leqslant t} E\left|x\left(s \wedge \tau_{k}\right)\right|^{p} \leqslant \frac{k_{0}}{1-k_{0}} E\|\xi\|^{p}+\frac{1}{\left(1-k_{0}\right)^{p}} \sup _{0 \leqslant s \leqslant t} E\left|x\left(s \wedge \tau_{k}\right)-D\left(x_{s \wedge \tau_{k}}\right)\right|^{p} .
$$

By (10) and (12), we have

$$
\begin{aligned}
\sup _{0 \leqslant s \leqslant t} E\left|x\left(s \wedge \tau_{k}\right)\right|^{p} \leqslant & \frac{k_{0}}{1-k_{0}} E\|\xi\|^{p}+\frac{M}{c_{1}\left(1-k_{0}\right)^{p}} \\
& +\frac{1}{c_{1}\left(1-k_{0}\right)^{p}} \sup _{0 \leqslant s \leqslant t} E \int_{0}^{s \wedge \tau_{k}} K(\rho+\tau)[1+2 V(\rho, x(\rho))] d \rho .
\end{aligned}
$$


Applying $c_{1}|x|^{p} \leqslant V(x, t) \leqslant c_{2}|x|^{p}$ once again and by inequality (13), we can obtain

$$
\begin{aligned}
\sup _{0 \leqslant s \leqslant t} E V\left(s \wedge \tau_{k}, x\left(s \wedge \tau_{k}\right)\right) \leqslant & c_{3}+\frac{c_{2}}{c_{1}\left(1-k_{0}\right)^{p}} \sup _{0 \leqslant s \leqslant t} E \int_{0}^{s \wedge \tau_{k}} K(\rho+\tau)[1+2 V(\rho, x(\rho))] d \rho \\
& \leqslant c_{3}+\frac{c_{2}}{c_{1}\left(1-k_{0}\right)^{p}} \sup _{0 \leqslant s \leqslant t} E \int_{0}^{s} K(\rho+\tau)\left[1+2 V\left(\rho \wedge \tau_{k}, x\left(\rho \wedge \tau_{k}\right)\right)\right] d \rho
\end{aligned}
$$

where $c_{3}=\frac{c_{2} k_{0}}{1-k_{0}} E\|\xi\|^{p}+\frac{c_{2} M}{c_{1}\left(1-k_{0}\right)^{p}}$.

And inequality (14) implies that

$$
\sup _{0 \leqslant s \leqslant t} E V\left(x\left(s \wedge \tau_{k}\right), s \wedge \tau_{k}\right) \leqslant c_{3}+\frac{2 c_{2}}{c_{1}\left(1-k_{0}\right)^{p}} \int_{0}^{t} K(\rho+\tau)\left[0.5+\sup _{0 \leqslant \beta \leqslant \rho} E V\left(\beta \wedge \tau_{k}, x\left(\beta \wedge \tau_{k}\right)\right)\right] d \rho
$$

By the Gronwall inequality [14], we therefore obtain

$$
0.5+E V\left(x\left(t \wedge \tau_{k}\right), t \wedge \tau_{k}\right) \leqslant\left(0.5+c_{3}\right) e^{\frac{2 c_{2}}{c_{1}\left(1-k_{0}\right)^{p}} \int_{0}^{t} K(\rho+\tau) d \rho} .
$$

Consequently,

$$
\begin{aligned}
c_{1} k^{p} P\left(\tau_{k} \leqslant t\right) & \left.\leqslant c_{1} E\left(\left|x\left(\tau_{k}\right)\right|^{p} I_{\{} \tau_{k} \leqslant t\right\}\right) \leqslant c_{1} E\left|x\left(t \wedge \tau_{k}\right)\right|^{p} \\
& \leqslant E V\left(x\left(t \wedge \tau_{k}\right), t \wedge \tau_{k}\right) \leqslant\left(0.5+c_{3}\right) e^{\frac{2 c_{2}}{c_{1}\left(1-k_{0}\right)^{p}} \int_{0}^{t} K(\rho+\tau) d \rho} .
\end{aligned}
$$

Letting $k \rightarrow \infty$ in the above inequality, then we can obtain $\lim _{k \rightarrow \infty} P\left(\tau_{k} \leqslant t\right)=0$. Since $t$ is arbitrary, we have $P\left(\tau_{k}<\infty\right)=0$. Hence, $\tau_{\infty}=\infty$ a.s. And by inequality (15), we have

$$
E V(t, x(t)) \leqslant\left(0.5+c_{3}\right) e^{\frac{2 c_{2}}{c_{1}\left(1-k_{0}\right)^{p}} \int_{0}^{t} K(\rho+\tau) d \rho} .
$$

The proof is completed.

\section{Boundedness and almost sure exponential stability for solution}

In this section, with the notations introduced in the previous section, we discuss the boundedness of the $p$ th moment of the solution. Moreover, if $x(t)=0$ is the trivial solution, we can also obtain that the trivial solution is almost surly exponentially stable under certain conditions.

Definition 2. (Almost sure exponential stability) If $f(t, 0)=h(u, 0)=D(0) \equiv 0$ for $t \geqslant 0, u \in U$. Then the trivial solution of equation (1) is said to be almost surely exponentially stable if

$$
\limsup _{t \rightarrow \infty} \frac{1}{t} \log |x(t ; 0, \xi)| \leqslant 0 \quad \text { a.s. }
$$

for all $\xi \in R^{n}$.

Theorem 2. Let Assumptions 1,2,3 hold except (3) which is replaced by

$$
\begin{aligned}
\mathcal{L} V(t, \phi) \leqslant & -\alpha_{1} V(t, \phi(0))+\alpha_{2} \int_{-\tau}^{0} V(t+\theta, \phi(\theta)) d \mu(\theta) \\
& -H(t, \phi(0))+\alpha_{3} \int_{-\tau}^{0} H(t+\theta, \phi(\theta)) d \nu(\theta),
\end{aligned}
$$

where $\alpha_{1}>\alpha_{2} \geqslant 0$ and $\alpha_{3} \in(0,1)$. Then for any given initial data $\xi$, there is a unique global solution $x(t)$ to equation (1) which has the property that

$$
E|x(t)|^{p} \leqslant \frac{\frac{C\left(1-k_{0}\right)^{1-p}}{c_{1}}+k_{0} E\|\xi\|^{p}}{1-k_{0}}
$$


for any $t \geqslant 0$. Where $C=V\left(0, x(0)-D\left(x_{0}\right)\right)+\alpha_{2} e^{\varepsilon \tau} E \int_{-\tau}^{0} V(s, x(s)) d s+\alpha_{3} e^{\varepsilon \tau} \int_{-\tau}^{0} H(s, x(s)) d s$ while $\varepsilon=\varepsilon_{1} \wedge \varepsilon_{2}$. And $\varepsilon_{1}, \varepsilon_{2}$ satisfy

$$
\begin{aligned}
& \alpha_{1}-\alpha_{2} e^{\varepsilon_{1} \tau}=0, \\
& 1-\alpha_{3} e^{\varepsilon_{2} \tau}=0,
\end{aligned}
$$

respectively. Moreover,

$$
\int_{0}^{\infty} H(t, x(t)) d t \leqslant \frac{M}{1-\alpha_{3}} .
$$

where $M=E\left(V\left(0, x(0)-D\left(x_{0}\right)\right)+\alpha_{2} \int_{-\tau}^{0} V(s, x(s)) d s+\alpha_{3} \int_{-\tau}^{0} H(s, x(s)) d s\right)$.

Proof. First, we can observe that (16) is stronger than (3). So, we can obtain that there is a unique global solution $x(t)$ to equation (1) by Theorem 3.1. By Itô formula and inequality (16), we can compute

$$
\begin{aligned}
& E\left(e^{\varepsilon\left(t \wedge \tau_{k}\right)} V\left(t \wedge \tau_{k}, x\left(t \wedge \tau_{k}\right)-D\left(x_{t \wedge \tau_{k}}\right)\right)-V\left(0, x(0)-D\left(x_{0}\right)\right)\right. \\
& =E \int_{0}^{t \wedge \tau_{k}} \varepsilon e^{\varepsilon s} V\left(s, x(s)-D\left(x_{s}\right)\right) d s+E \int_{0}^{t \wedge \tau_{k}} e^{\varepsilon s} \mathcal{L} V\left(s, x_{s}\right) d s \\
& \leqslant E \int_{0}^{t \wedge \tau_{k}} \varepsilon e^{\varepsilon s} V\left(s, x(s)-D\left(x_{s}\right)\right) d s-\alpha_{1} E \int_{0}^{t \wedge \tau_{k}} e^{\varepsilon s} V(s, x(s)) d s \\
& +\alpha_{2} E \int_{0}^{t \wedge \tau_{k}} \int_{-\tau}^{0} e^{\varepsilon s} V(s+\theta, x(s+\theta)) d \mu(\theta) d s-E \int_{0}^{t \wedge \tau_{k}} e^{\varepsilon s} H(s, x(s)) d s \\
& +\alpha_{3} E \int_{0}^{t \wedge \tau_{k}} \int_{-\tau}^{0} e^{\varepsilon s} H(s+\theta, x(s+\theta)) d \nu(\theta) d s \\
& \leqslant E \int_{0}^{t \wedge \tau_{k}} \varepsilon e^{\varepsilon s} V\left(s, x(s)-D\left(x_{s}\right)\right) d s-\alpha_{1} E \int_{0}^{t \wedge \tau_{k}} e^{\varepsilon s} V(s, x(s)) d s \\
& +\alpha_{2} E \int_{-\tau}^{t \wedge \tau_{k}} e^{\varepsilon(s+\tau)} V(s, x(s)) d s-E \int_{0}^{t \wedge \tau_{k}} e^{\varepsilon s} H(s, x(s)) d s \\
& +\alpha_{3} E \int_{-\tau}^{t \wedge \tau_{k}} e^{\varepsilon(s+\tau)} H(s, x(s)) d s
\end{aligned}
$$

for any $t \geqslant 0$ and $\tau_{k}$ is the same defined as theorem 3.1. We take $\varepsilon=\varepsilon_{1} \wedge \varepsilon_{2}$, and $\varepsilon_{1}, \varepsilon_{2}$ satisfy

$$
\begin{aligned}
& \alpha_{1}-\alpha_{2} e^{\varepsilon_{1} \tau}=0 \\
& 1-\alpha_{3} e^{\varepsilon_{2} \tau}=0
\end{aligned}
$$

respectively. Then the inequality (19) leads to

$$
\begin{aligned}
& E\left(e^{\varepsilon\left(t \wedge \tau_{k}\right)}\right) V\left(t \wedge \tau_{k}, x\left(t \wedge \tau_{k}\right)-D\left(x_{t \wedge \tau_{k}}\right)\right) \leqslant \\
& V\left(0, x(0)-D\left(x_{0}\right)\right)+\alpha_{2} e^{\varepsilon \tau} E \int_{-\tau}^{0} V(s, x(s)) d s+\alpha_{3} e^{\varepsilon \tau} E \int_{-\tau}^{0} H(s, x(s)) \\
& \quad+\varepsilon E \int_{0}^{t \wedge \tau_{k}} e^{\varepsilon s} V\left(s, x(s)-D\left(x_{s}\right)\right) d s=C+\varepsilon E \int_{0}^{t \wedge \tau_{k}} e^{\varepsilon s} V\left(s, x(s)-D\left(x_{s}\right)\right) d s
\end{aligned}
$$

where $C=V\left(0, x(0)-D\left(x_{0}\right)\right)+\alpha_{2} e^{\varepsilon \tau} E \int_{-\tau}^{0} V(s, x(s)) d s+\alpha_{3} e^{\varepsilon \tau} E \int_{-\tau}^{0} H(s, x(s))$.

And by inequality (20) and Fubini theorem [15], we can obtain

$$
\begin{aligned}
& E\left(e^{\varepsilon\left(t \wedge \tau_{k}\right)}\right) V\left(t \wedge \tau_{k}, x\left(t \wedge \tau_{k}\right)-D\left(x_{t \wedge \tau_{k}}\right)\right) \\
& \leqslant C+\varepsilon E \int_{0}^{t} e^{\varepsilon\left(s \wedge \tau_{k}\right)} V\left(s \wedge \tau_{k}, x\left(s \wedge \tau_{k}\right)-D\left(x_{s \wedge \tau_{k}}\right)\right) d s \\
& =C+\varepsilon \int_{0}^{t} E\left(e^{\varepsilon\left(s \wedge \tau_{k}\right)} V\left(s \wedge \tau_{k}, x\left(s \wedge \tau_{k}\right)-D\left(x_{s \wedge \tau_{k}}\right)\right)\right) d s,
\end{aligned}
$$


hence, using the Gronwall inequality [14], we derive that

$$
E\left(e^{\varepsilon\left(t \wedge \tau_{k}\right)}\right) V\left(t \wedge \tau_{k}, x\left(t \wedge \tau_{k}\right)-D\left(x_{t \wedge \tau_{k}}\right)\right) \leqslant C e^{\varepsilon t} .
$$

Letting $k \rightarrow \infty$, we obtain that

$$
E\left(V\left(t, x(t)-D\left(x_{t}\right)\right)\right) \leqslant C .
$$

By Assumption 2 and inequality (21), we know that

$$
c_{1} E\left|x(t)-D\left(x_{t}\right)\right|^{p} \leqslant E\left(V\left(t, x(t)-D\left(x_{t}\right)\right)\right) \leqslant C .
$$

Recall the fundamental inequality : $|a+b|^{p} \leqslant(1-k)^{1-p}|a|^{p}+k^{1-p}|b|^{p}$ for any $a, b \in R, p \geqslant 1$, $k \in(0,1)$. Then,

$$
\left|x(t)-D\left(x_{t}\right)\right|^{p} \geqslant \frac{|x(t)|^{p}-k_{0}^{1-p}\left|D\left(x_{t}\right)\right|^{p}}{\left(1-k_{0}\right)^{1-p}} .
$$

Substituting (23) into (22) yields

$$
E|x(t)|^{p} \leqslant \frac{C\left(1-k_{0}\right)^{1-p}}{c_{1}}+k_{0}^{1-p} E\left|D\left(x_{t}\right)\right|^{p} .
$$

By Assumption 3 and the inequality (24), we therefore obtain

$$
\begin{aligned}
\sup _{0 \leqslant s \leqslant t} E|x(s)|^{p} & \leqslant \frac{C\left(1-k_{0}\right)^{1-p}}{c_{1}}+k_{0}^{1-p} \sup _{0 \leqslant s \leqslant t} E\left|D\left(x_{s}\right)\right|^{p} \\
& \leqslant \frac{C\left(1-k_{0}\right)^{1-p}}{c_{1}}+k_{0} \sup _{0 \leqslant s \leqslant t-\tau \leqslant \theta \leqslant 0} \sup _{-} E|x(s+\theta)|^{p} \\
& \leqslant \frac{C\left(1-k_{0}\right)^{1-p}}{c_{1}}+k_{0} \sup _{-\tau \leqslant s \leqslant t} E|x(s)|^{p} \\
& \leqslant \frac{C\left(1-k_{0}\right)^{1-p}}{c_{1}}+k_{0}\left(E\|\xi\|^{p}+\sup _{0 \leqslant s \leqslant t} E|x(s)|^{p}\right)
\end{aligned}
$$

for any $t \geqslant 0$, which yields

$$
E|x(t)|^{p} \leqslant \sup _{0 \leqslant s \leqslant t} E|x(s)|^{p} \leqslant \frac{\frac{C\left(1-k_{0}\right)^{1-p}}{c_{1}}+k_{0} E\|\xi\|^{p}}{1-k_{0}}
$$

and the assertion (17) follows.

To prove the other assertion (15), we apply Itô formula to $V(t, x)$ directly,

$$
\begin{aligned}
E V\left(t \wedge \tau_{k}, x\left(t \wedge \tau_{k}\right)-D\left(x_{t \wedge \tau_{k}}\right)\right) & =E V\left(0, x(0)-D\left(x_{0}\right)\right)+E \int_{0}^{t \wedge \tau_{k}} \mathcal{L} V\left(s, x_{s}\right) d s \\
& \leqslant E V\left(0, x(0)-D\left(x_{0}\right)\right)-\alpha_{1} E \int_{0}^{t \wedge \tau_{k}} V(s, x(s)) d s \\
& +\alpha_{2} E \int_{0}^{t \wedge \tau_{k}} \int_{-\tau}^{0} V(s+\theta, x(s+\theta)) d \mu(\theta) d s \\
& +\alpha_{3} E \int_{0}^{t \wedge \tau_{k}} \int_{-\tau}^{0} H(s+\theta, x(s+\theta)) d \nu(\theta) d s \\
& -E \int_{0}^{t \wedge \tau_{k}} H(s, x(s)) d s .
\end{aligned}
$$

By Fubini theorem [15], we can compute

$$
\begin{aligned}
\int_{0}^{t \wedge \tau_{k}} \int_{-\tau}^{0} V(s+\theta, x(s+\theta)) d \mu(\theta) d s & =\int_{-\tau}^{0} \int_{0}^{t \wedge \tau_{k}} V(s+\theta, x(s+\theta)) d s d \mu(\theta) \\
& \leqslant \int_{-\tau}^{0}\left(\int_{0}^{t \wedge \tau_{k}} V(s, x(s)) d s\right) d \mu(\theta) \\
& \leqslant \int_{-\tau}^{t \wedge \tau_{k}} V(s, x(s)) d s .
\end{aligned}
$$


Similarly,

$$
\int_{0}^{t \wedge \tau_{k}} \int_{-\tau}^{0} H(s+\theta, x(s+\theta)) d \mu(\theta) d s \leqslant \int_{-\tau}^{t \wedge \tau_{k}} H(s, x(s)) d s
$$

Substituting (26) and (27) into (25), we obtain

$$
\begin{aligned}
E V\left(t \wedge \tau_{k}, x\left(t \wedge \tau_{k}\right)-D\left(x_{t \wedge \tau_{k}}\right)\right) & \leqslant M+\left(\alpha_{2}-\alpha_{1}\right) E \int_{0}^{t \wedge \tau_{k}} V(s, x(s)) d s \\
& +\left(\alpha_{3}-1\right) E \int_{0}^{t \wedge \tau_{k}} H(s, x(s)) d s \\
& \leqslant M+\left(\alpha_{3}-1\right) E \int_{0}^{t \wedge \tau_{k}} H(s, x(s)) d s
\end{aligned}
$$

where $M=E\left(V\left(0, x(0)-D\left(x_{0}\right)\right)+\alpha_{2} \int_{-\tau}^{0} V(s, x(s)) d s+\alpha_{3} \int_{-\tau}^{0} H(s, x(s)) d s\right)$. Since $\alpha_{3} \in(0,1)$ and $V(t, x) \geqslant c_{1}|x|^{p} \geqslant 0$, the inequality (28) yields the assertion (18).

Remark 1. Compared with the other known results, such that $[9,11,12]$, the right side of the inequality (16) is short of a positive constant. The reason is that our system is a neutral system, we cannot obtain the same result if there is a positive constant in the right hand of the inequality (16) by our current technique. That is, we can roughly explain that the positive constant can "compensate" the neutral system.

We can obtain the upper bound of the pth moment of the solution from Theorem 4.1. Next, we will prove that if there is a trivial solution, then the boundedness of the $p$ th moment of the solution implies the trivial solution is almost surely exponentially stable.

Theorem 3. Let all the assumptions of Theorem 4.1 hold and $f(t, 0)=h(u, 0)=D(0) \equiv 0$ for $t \geqslant 0, u \in U$. Then the trivial solution to the equation (1) is almost surely exponentially stable. That is, the unique global solution $x(t)$ has the property that

$$
\limsup _{t \rightarrow \infty} \frac{1}{t} \log |x(t ; 0, \xi)| \leqslant 0 \quad \text { a.s. }
$$

for all $\xi \in R^{n}$.

Proof. If $f(t, 0)=h(u, 0)=D(0) \equiv 0$ for $t \geqslant 0, u \in U$, then the equation admits a trivial solution $x(t ; 0) \equiv 0$ corresponding to the initial data $x_{0}=0$.

For simplify, we set $M \triangleq \frac{\frac{C\left(1-k_{0}\right)^{1-p}}{c_{1}}+k_{0} E\|\xi\|^{p}}{1-k_{0}}$. Next, for each $n=1,2, \cdots$, and any $\epsilon>0$, it follows from Markov inequality [16] and Theorem 4.1 that

$$
P\left\{\omega:|x(t, \omega)|^{p}>e^{\epsilon n}\right\} \leqslant \frac{E|x(t)|^{p}}{e^{\epsilon n}} \leqslant M e^{-\epsilon n}
$$

for any $t \in[n-1, n]$.

Since $\sum_{n=0}^{\infty} M e^{-\epsilon n}<\infty$, by the Borel-Cantelli lemma [14], there is an integer $n_{0}$ such that

$$
|x(t)|^{p} \leqslant e^{\epsilon n} \quad \text { a.s. }
$$

for all $n \geqslant n_{0}$ and $t \in[n-1, n]$. Then, we can obtain

$$
\frac{1}{t} \log |x(t)|=\frac{1}{p t} \log |x(t)|^{p} \leqslant \frac{\epsilon n}{p(n-1)} .
$$

Letting $n \rightarrow \infty$ on both sides of inequality (25),

$$
\limsup _{t \rightarrow \infty} \frac{1}{t} \log |x(t)| \leqslant \frac{\epsilon}{p},
$$

which is the required assertion because $\epsilon>0$ is arbitrary. 
Theorem 4. Let Assumption 1,2,3 hold except (3) which is replaced by

$$
\begin{aligned}
\mathcal{L} V(t, \phi) \leqslant & -\alpha_{1} V(t, \phi(0))+\alpha_{2} \int_{-\tau}^{0} V(t+\theta, \phi(\theta)) d \mu(\theta) \\
& -H(t, \phi(0))+\alpha_{3} \int_{-\tau}^{0} H(t+\theta, \phi(\theta)) d \nu(\theta)-\alpha_{4} V(t, \phi(0)-D(\phi)),
\end{aligned}
$$

where $\alpha$ is a constant, and $\alpha_{1}>\alpha_{2} \geqslant 0, \alpha_{3} \in(0,1), \alpha_{4}>0$. Then for any given initial data $\xi$, there is a unique global solution $x(t)$ to equation (1) which has the property that

$$
\limsup _{t \rightarrow \infty} E|x(t)|^{p} \leqslant \frac{\alpha}{\varepsilon c_{1}\left(1-k_{0}\right)^{p}}+\frac{k_{0} E\|\xi\|^{p}}{1-k_{0}}
$$

for any $t \geqslant 0$.

Where $\xi$ is the initial date and $\varepsilon=\varepsilon_{1} \wedge \varepsilon_{2} \wedge \alpha_{4}$. And $\varepsilon_{1}, \varepsilon_{2}$ satisfy

$$
\begin{aligned}
& \alpha_{1}-\alpha_{2} e^{\varepsilon_{1} \tau}=0, \\
& 1-\alpha_{3} e^{\varepsilon_{2} \tau}=0,
\end{aligned}
$$

respectively.

If $f(t, 0)=h(u, 0)=D(0) \equiv 0$ for $t \geqslant 0, u \in U$. Then the trivial solution to the equation (1) is almost surely exponentially stable. That is, the unique global solution $x(t)$ has the property that

$$
\limsup _{t \rightarrow \infty} \frac{1}{t} \log |x(t ; 0, \xi)| \leqslant 0 \quad \text { a.s. }
$$

for all $\xi \in R^{n}$.

Proof. Similar to the proof of Theorem 4.1, we obtain

$$
\begin{aligned}
& E\left(e^{\varepsilon\left(t \wedge \tau_{k}\right)} V\left(t \wedge \tau_{k}, x\left(t \wedge \tau_{k}\right)-D\left(x_{t \wedge \tau_{k}}\right)\right)\right)-E V\left(0, x(0)-D\left(x_{0}\right)\right) \\
& =E \int_{0}^{t \wedge \tau_{k}} \varepsilon e^{\varepsilon s} V\left(s, x(s)-D\left(x_{s}\right)\right) d s+E \int_{0}^{t \wedge \tau_{k}} e^{\varepsilon s} \mathcal{L} V\left(s, x_{s}\right) d s \\
& \leqslant E \int_{0}^{t \wedge \tau_{k}} e^{\varepsilon s} V(s, x(s)) d s+E \int_{0}^{t \wedge \tau_{k}} \alpha e^{\varepsilon s} d s \\
& -\alpha_{1} E \int_{0}^{t \wedge \tau_{k}} e^{\varepsilon s} V(s, x(s)) d s+\alpha_{2} E \int_{0}^{t \wedge \tau_{k}} \int_{-\tau}^{0} e^{\varepsilon s} V(s+\theta, x(s+\theta)) d \mu(\theta) d s \\
& -E \int_{0}^{t \wedge \tau_{k}} e^{\varepsilon s} H(s, x(s)) d s+\alpha_{3} E \int_{0}^{t \wedge \tau_{k}} \int_{-\tau}^{0} e^{\varepsilon s} H(s+\theta, x(s+\theta)) d \nu(\theta) d s \\
& -\alpha_{4} E \int_{0}^{t \wedge \tau_{k}} e^{\varepsilon s} V\left(s, x(s)-D\left(x_{s}\right)\right) d s \\
& \leqslant \alpha_{2} e^{\varepsilon \tau} E \int_{-\tau}^{0} V(s, x(s)) d s+\alpha_{3} e^{\varepsilon \tau} E \int_{-\tau}^{0} H(s, x(s)) d s \\
& +\alpha \frac{e^{\varepsilon t}}{\varepsilon}-\left(\alpha_{4}-\varepsilon\right) E \int_{0}^{t \wedge \tau_{k}} e^{\varepsilon s} V\left(s, x(s)-D\left(x_{s}\right)\right) d s \\
& -\left(\alpha_{1}-\alpha_{2} e^{\varepsilon \tau}\right) E \int_{0}^{t \wedge \tau_{k}} e^{\varepsilon s} V(s, x(s)) d s-\left(1-\alpha_{3} e^{\varepsilon \tau}\right) E \int_{0}^{t \wedge \tau_{k}} e^{\varepsilon s} H(s, x(s)) d s \\
& \leqslant \alpha_{2} e^{\varepsilon \tau} E \int_{-\tau}^{0} V(s, x(s)) d s+\alpha_{3} e^{\varepsilon \tau} E \int_{-\tau}^{0} H(s, x(s)) d s+\alpha \frac{e^{\varepsilon t}}{\varepsilon} . \\
&
\end{aligned}
$$

Taking $k \rightarrow \infty$ on both sides, then we get

$$
E\left(e^{\varepsilon t} V\left(t, x(t)-D\left(x_{t}\right)\right) \leqslant R+\frac{\alpha e^{\varepsilon t}}{\varepsilon},\right.
$$


where $R=E V\left(0, x(0)-D\left(x_{0}\right)\right)+e^{\varepsilon \tau} E \int_{-\tau}^{0} V(s, x(s)) d s+\alpha_{3} e^{\varepsilon \tau} E \int_{-\tau}^{0} H(s, x(s)) d s$ is a constant. By inequality (34), we can compute

$$
E V\left(t, x(t)-D\left(x_{t}\right)\right) \leqslant R e^{-\varepsilon t}+\frac{\alpha}{\varepsilon} .
$$

Using the following two inequalities, Assumption 3 and inequality (34)

$$
\begin{aligned}
& c_{1}\left|x(t)-D\left(x_{t}\right)\right|^{p} \leqslant V\left(t, x(t)-D\left(x_{t}\right)\right) \\
& |x(t)|^{p} \leqslant\left(1-k_{0}\right)^{1-p}\left|x(t)-D\left(x_{t}\right)\right|^{p}+k_{0}^{1-p}\left|D\left(x_{t}\right)\right|^{p},
\end{aligned}
$$

we can derive

$$
\begin{aligned}
E|x(t)|^{p} & \leqslant \frac{\left(1-k_{0}\right)^{1-p}}{c_{1}} E V\left(t, x(t)-D\left(x_{t}\right)\right)+k_{0}^{1-p} E\left|D\left(x_{t}\right)\right|^{p} \\
& \leqslant \frac{\left(1-k_{0}\right)^{1-p}}{c_{1}}\left(R e^{-\varepsilon t}+\frac{\alpha}{\varepsilon}\right)+k_{0} \sup _{-\tau \leqslant \theta \leqslant 0} E|x(t+\theta)|^{p}
\end{aligned}
$$

for any $t \geqslant 0$. Then

$$
\begin{aligned}
\sup _{0 \leqslant s \leqslant t} E|x(s)|^{p} \leqslant & \frac{\left(1-k_{0}\right)^{1-p}}{c_{1}}\left(R e^{-\varepsilon t}+\frac{\alpha}{\varepsilon}\right)+k_{0} \sup _{0 \leqslant s \leqslant t-\tau \leqslant \theta \leqslant 0} E|x(s+\theta)|^{p} \\
& \leqslant \frac{\left(1-k_{0}\right)^{1-p}}{c_{1}}\left(R e^{-\varepsilon t}+\frac{\alpha}{\varepsilon}\right)+k_{0} \sup _{-\tau \leqslant s \leqslant t} E|x(s)|^{p} \\
& \leqslant \frac{\left(1-k_{0}\right)^{1-p}}{c_{1}}\left(R e^{-\varepsilon t}+\frac{\alpha}{\varepsilon}\right)+k_{0} E\|\xi\|^{p}+k_{0} \sup _{0 \leqslant s \leqslant t} E|x(s)|^{p},
\end{aligned}
$$

and inequality (36) implies

$$
E|x(t)|^{p} \leqslant \sup _{0 \leqslant s \leqslant t} E|x(s)|^{p} \leqslant \frac{R e^{-\varepsilon t}+\frac{\alpha}{\varepsilon}}{c_{1}\left(1-k_{0}\right)^{p}}+\frac{k_{0} E\|\xi\|^{p}}{1-k_{0}} .
$$

Consequently, we can obtain

$$
\limsup _{t \rightarrow \infty} E|x(t)|^{p} \leqslant \frac{\alpha}{\varepsilon c_{1}\left(1-k_{0}\right)^{p}}+\frac{k_{0} E\|\xi\|^{p}}{1-k_{0}}
$$

which is the assertion (30). The proof of the assertion (33) is similar to the proof of Theorem 4.2.

Remark 2. Note that the right hand of the inequality (30) contains a constant $\alpha$. That is because the right side of the inequality also include another negative term $-\alpha_{4} V(t, \phi(0)-D(\phi))$, which can "compensate" the constant. Moreover, if $\alpha \leqslant 0$, we can obtain that the $p$ th moment of the solution is stable.

\section{$5 \quad$ Examples}

Example 1. Consider the following equation

$$
d x(t)=\left(-\frac{1}{2} x^{2}(t)+t \int_{-1}^{0} x(t+\theta) d \theta\right) d t+\sqrt{t} \int_{-1}^{0} x(t+\theta) d \theta d N(t) .
$$

In this example, we set $\tau=1, U=\{1\}$. Define

$$
\begin{aligned}
& D(\varphi)=0 \\
& f(t, \varphi)=-\frac{1}{2} x^{2}(t)+t \int_{-1}^{0} \varphi(\theta) d \theta \\
& h(1, \varphi)=\sqrt{t} \int_{-1}^{0} \varphi(\theta) d \theta .
\end{aligned}
$$


Choose $V(t, x)=x^{2}$, by Itô formula and Hölder inequality [14], we can compute

$$
\begin{aligned}
\mathcal{L} V(t, \varphi) & =2 \varphi(0)\left(-\frac{1}{2} \varphi^{2}(0)+t \int_{-1}^{0} \varphi(\theta) d \theta\right)+\left(\varphi(0)+\sqrt{t} \int_{-1}^{0} \varphi(\theta) d \theta\right)^{2} \\
& -\varphi^{2}(0)-2 \sqrt{t} \varphi(0) \int_{-1}^{0} \varphi(\theta) d \theta \\
& =-\varphi^{3}(0)+2 t \varphi(0) \int_{-1}^{0} \varphi(\theta) d \theta+t\left(\int_{-1}^{0} \varphi(\theta) d \theta\right)^{2} \\
& \leqslant 2 t\left[\varphi^{2}(0)+\int_{-1}^{0} \varphi^{2}(\theta) d \theta\right]-\varphi^{3}(0) .
\end{aligned}
$$

Taking $K(t)=2 t$, then we can conclude that the equation (37) has a unique global solution for any given initial data by Theorem 3.1 .

Remark 3. Example 1 have checked the conclusion of the Theorem 3.1. Next example will mainly show the effectiveness of the Theorem 4.1.

Example 2. Let us consider the equation

$$
d\left[x(t)-\frac{2}{3} \int_{-1}^{0} x(t+\theta) d \theta\right]=\left(-x(t)-x^{3}(t)\right) d t+\frac{1}{2} \int_{-1}^{0} x(t+\theta) d \theta d N(t) .
$$

In this example, we set $U=\{1\}$ and define

$$
\begin{aligned}
& D(\varphi)=\frac{2}{3} \int_{-1}^{0} \varphi(\theta) d \theta, \\
& F(t, \varphi)=-\varphi(0)-\varphi^{3}(0), \\
& h(1, \varphi)=\frac{1}{2} \int_{-1}^{0} \varphi(\theta) d \theta .
\end{aligned}
$$

for $t \geqslant 0, \varphi \in D([-1,0] ; R)$. Then the coefficients satisfy Assumption 1 . If we choose $p=2$, the hölder inequality [14] yields

$$
E|D(\varphi)|^{2}=\frac{4}{9} E\left|\int_{-1}^{0} \varphi(\theta) d \theta\right|^{2} \leqslant \frac{4}{9} \sup _{-1 \leqslant \theta \leqslant 0} E|\varphi(\theta)|^{2},
$$

which implies Assumption 3.

Define $V(x)=|x|^{2}$ and employing Itô formula, Young inequality [17] and Hölder inequality [14], we can compute

$$
\begin{aligned}
\mathcal{L} V(t, \varphi) & =2\left(\varphi(0)-\frac{2}{3} \int_{-1}^{0} \varphi(\theta) d \theta\right)\left(-\varphi(0)-\varphi^{3}(0)\right) \\
& +\left(\varphi(0)-\frac{2}{3} \int_{-1}^{0} \varphi(\theta) d \theta+\frac{1}{2} \int_{-1}^{0} \varphi(\theta) d \theta\right)^{2}-\left(\varphi(0)-\frac{2}{3} \int_{-1}^{0} \varphi(\theta) d \theta\right)^{2} \\
& -\left(\varphi(0)-\frac{2}{3} \int_{-1}^{0} \varphi(\theta) d \theta\right) \int_{-1}^{0} \varphi(\theta) d \theta \\
& \leqslant-\frac{4}{3}|\varphi(0)|^{2}+\frac{11}{12}\left|\int_{-1}^{0} \varphi(\theta) d \theta\right|^{2}-|\varphi(0)|^{4}+\frac{1}{3}\left|\int_{-1}^{0} \varphi(\theta) d \theta\right|^{4} \\
& \leqslant-\frac{4}{3} \varphi^{2}(0)+\frac{11}{12} \int_{-1}^{0} \varphi^{2}(\theta) d \theta-\varphi^{4}(0)+\frac{1}{3} \int_{-1}^{0} \varphi^{4}(\theta) d \theta .
\end{aligned}
$$

Applying Theorem 3.1, if we choose $H(t, x)=|x|^{4}$, then we can obtain that there is a unique global solution for any given initial data. The conditions in Theorem 4.1 are satisfied when we choose $\alpha_{1}=$ $\frac{4}{3}, \alpha_{2}=\frac{11}{12}, \alpha_{3}=\frac{1}{3}$. Therefore, if we take $\xi(\theta)=\theta+1,-1 \leqslant \theta \leqslant 0$, then we can compute $C=0.986$ and $E|x(t)|^{2} \leqslant 4.194$.

Particularly, the trivial solution $x(t) \equiv 0$ of equation (38) is almost surely exponentially stable in theory. Moreover, it can be seen from Figure 1 that the trivial solution is stable. 


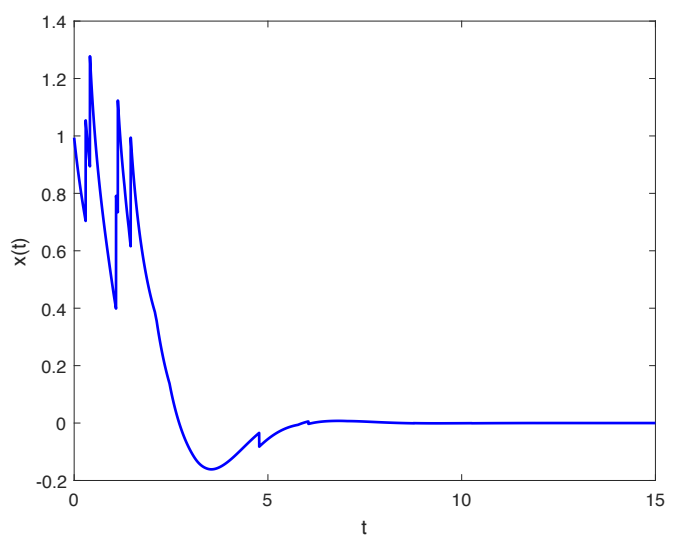

Figure 1 Trajectory of state

\section{Conclusion}

In this paper, the existence and uniqueness of solution of the neutral stochastic functional differential equations with pure jumps under the local Lipschitz condition and the Khasminskii-type condition has been solved. There was no need for the linear growth condition, so we could deal with the problems that the coefficients of the equation are of highly order. Moreover, we obtained the boundness of $p$ th moment of the solution. And if there was a trivial solution for the equation, we also proved that the trivial solution is almost surely exponentially stable.

Acknowledgements This work was supported by the National Natural Science Foundation of China under Grants 61573156, 61273126, 61503142, the Ph.D. Start-up Fund of Natural Science Foundation of Guangdong Province under Grant 2014A030310388, and the Fundamental Research Funds for the Central Universities under Grant x2zdD2153620.

Conflict of interest The authors declare that they have no conflict of interest.

\section{References}

1 L. Wu, W. X. Zheng, H. Gao, Dissipativity-based sliding mode control of switched stochastic systems, IEEE Transactions on Automatic Control 58 (3) (2013) 785-791.

2 B. Zhang, F. Deng, X. Zhao, C. Zhang, Hybrid control of stochastic chaotic system based on memristive lorenz system with discrete and distributed time-varying delays, IET Control Theory \& Applications.

3 D. Wu, X. Luo, S. Zhu, Stochastic system with coupling between non-gaussian and gaussian noise terms, Physica A: Statistical Mechanics and its Applications 373 (2007) 203-214.

4 J. Shao, C. Yuan, Transportation-cost inequalities for diffusions with jumps and its application to regime-switching processes, Journal of Mathematical Analysis and Applications 425 (2) (2015) 632-654.

5 Lee, Suzanne. S, Mykland, Per. A, Jumps in financial markets: A new nonparametric test and jump dynamics, Review of Financial studies 21 (6) (2008) 2535-2563.

6 R. J. Elliott, C.-J. U. Osakwe, Option pricing for pure jump processes with markov switching compensators, Finance and Stochastics 10 (2) (2006) 250-275.

7 W. Mao, Q. Zhu, X. Mao, Existence, uniqueness and almost surely asymptotic estimations of the solutions to neutral stochastic functional differential equations driven by pure jumps, Applied Mathematics and Computation 254 (2015) $252-265$.

8 L. Baosheng, H. Shigeng, F. Yang, Stochastic delay lotka-volterra model, Journal of Inequalities and Applications 2011 (1) (2011) 1.

9 M. Song, L. Hu, X. Mao, L. Zhang, Khasminskii-type theorems for stochastic functional differential equations, Discrete Contin. Dyn. Syst. Ser. B 18 (6) (2013) 1697-1714.

10 Q. Luo, X. Mao, Y. Shen, Generalised theory on asymptotic stability and boundedness of stochastic functional differential equations, Automatica 47 (9) (2011) 2075-2081.

11 F. Wu, S. Hu, Khasminskii-type theorems for stochastic functional differential equations with infinite delay, Statistics 
\& Probability Letters 81 (11) (2011) 1690-1694.

12 X. Mao, M. J. Rassias, Khasminskii-type theorems for stochastic differential delay equations, Stochastic Analysis and Applications 23 (5) (2005) 1045-1069.

13 D. Applebaum, Lévy processes and stochastic calculus, Cambridge university press, 2009.

14 X. Mao, Stochastic differential equations and applications, Elsevier, 2007.

15 P. A. Meyer, Probability and potentials, Vol. 357, Blaisdell Waltham, 1966.

16 W. Pawłucki, W. Pleśniak, Markov's inequality andc functions on sets with polynomial cusps, Mathematische Annalen 275 (3) (1986) 467-480.

17 W. Beckner, Inequalities in fourier analysis, Annals of Mathematics (1975) 159-182. 